\title{
Physicochemical Characterization Cascade of Nanoadjuvant-Antigen Systems for Improving Vaccines
}

\author{
Giuditta Guerrini ${ }^{1}{ }^{(\mathbb{D}}$, Antonio Vivi ${ }^{1}$, Sabrina Gioria ${ }^{2}$, Jessica Ponti ${ }^{2}$, Davide Magrì ${ }^{2}{ }^{\mathbb{D}}$, Arnd Hoeveler ${ }^{2}$, \\ Donata Medaglini ${ }^{1}$ and Luigi Calzolai ${ }^{2, *}$ \\ 1 Laboratory of Molecular Microbiology and Biotechnology, Department of Medical Biotechnologies, \\ University of Siena, 53100 Siena, Italy; guerrini12@student.unisi.it (G.G.); antonio.vivi@unisi.it (A.V.); \\ donata.medaglini@unisi.it (D.M.) \\ 2 European Commission, Joint Research Centre (JRC), 21027 Ispra, Italy; Sabrina.GIORIA@ec.europa.eu (S.G.); \\ Jessica.PONTI@ec.europa.eu (J.P.); Davide.MAGRI@ec.europa.eu (D.M.); Arnd.Hoeveler@ec.europa.eu (A.H.) \\ * Correspondence: luigi.calzolai@ec.europa.eu
}

\section{check for} updates

Citation: Guerrini, G.; Vivi, A.; Gioria, S.; Ponti, J.; Magrì, D.; Hoeveler, A.; Medaglini, D.; Calzolai, L. Physicochemical Characterization Cascade of Nanoadjuvant-Antigen Systems for Improving Vaccines. Vaccines 2021, 9, 544. https:/ / doi.org/10.3390/vaccines9060544

Academic Editors:

Eduardo Gomez-Casado and Amine A. Kamen

Received: 10 April 2021

Accepted: 17 May 2021

Published: 21 May 2021

Publisher's Note: MDPI stays neutral with regard to jurisdictional claims in published maps and institutional affiliations.

Copyright: (c) 2021 by the authors. Licensee MDPI, Basel, Switzerland. This article is an open access article distributed under the terms and conditions of the Creative Commons Attribution (CC BY) license (https:// creativecommons.org/licenses/by/ $4.0 /)$.

\begin{abstract}
Adjuvants have been used for decades to enhance the immune response to vaccines, in particular for the subunit-based adjuvants. Physicochemical properties of the adjuvant-protein antigen complexes, such as size, morphology, protein structure and binding, influence the overall efficacy and safety of the vaccine. Here we show how to perform an accurate physicochemical characterization of the nanoaluminum-ovalbumin complex. Using a combination of existing techniques, we developed a multi-staged characterization strategy based on measurements of increased complexity. This characterization cascade has the advantage of being very flexible and easily adaptable to any adjuvant-protein antigen combinations. It will contribute to control the quality of antigen-adjuvant complexes and immunological outcomes, ultimately leading to improved vaccines.
\end{abstract}

Keywords: adjuvants; antigens; characterization; vaccines

\section{Introduction}

Adjuvants are used in vaccines to enhance the immunogenicity of the antigen and are particularly important in the case of subunit vaccines where the recombinant protein antigens are poorly immunogenic and adjuvants are added as nonspecific immunostimulants to increase the efficacy of the vaccine formulation [1]. Among them, aluminum-based adjuvants have been used since 1932 in licensed human vaccines and, for nearly seven decades, they have been the only adjuvants authorized for human vaccines [2]. Their long-term success is mainly due to their safety profile, relatively low cost, and capacity to bind a large variety of subunit antigens [3]. The two types of aluminum adjuvants normally used in licensed vaccines are aluminum hydroxide (AH) and aluminum phosphate (AP), which can bind negatively or positively charged antigens. In addition, the surface charge of these adjuvants is $\mathrm{pH}$-dependent, and their capacity of binding protein antigens can be fine-tuned by changing either the $\mathrm{pH}$ or the ionic composition of the buffer in which the adjuvants and/or the antigens are used.

Remarkably, despite the significant improvement in the formulation of new vaccines, aluminum-based vaccines are still used due to their ability to cover safety requirements with simple and inexpensive formulations. Aluminum-based vaccines have also been developed against COVID-19. For example, Adimmune is developing a vaccine containing the recombinant receptor-binding domain (RBD) of SARS-CoV-2 spike protein with aluminum adjuvant, which is now in clinical trials [4,5].

Several studies demonstrated the mechanism of immunopotentiation carried out by aluminum, from the enhanced recruitment of antigen-presenting cells (APC) at the injection site, to the improved antigen uptake and maturation of APC [6]. 
According to the World Health Organization (WHO), the mechanism of action of aluminum-based adjuvants is still not completely understood; according to the World Health Organization (WHO), at least $80 \%$ of the antigen should be bound to the adjuvant itself. This allows the antigen to remain localized at the injection site for prolonged time, avoiding its spreading and dispersion in the interstitial fluids [7].

In addition to the physicochemical properties of the adjuvant, a key role is played by the nature of the interaction between adjuvant and antigen. To date, a comprehensive understanding of the physicochemical properties of the aluminum-antigen complex is lacking. Normally, electrostatic attraction, hydrophilic interaction, and ligand exchange occurs between antigen and adjuvant, rather than a covalent bond [8].

The stability of the recombinant antigen is a key aspect during the development of subunit vaccines. In fact, the conformational stability of antigens has a strong impact on immunogenicity and immune polarization. For example, proteolytic degradation of the protein is required before antigenic peptides are loaded on significant histocompatibility complex receptors. The structural stability of the protein influence the kinetics of the degradation (and ultimately its immunogenicity [9]), while the absorption of proteins on the adjuvant surface may also affect the protein structure and stability $[10,11]$. Thus, when developing "new subunit vaccines", it is important to characterize the whole proteinadjuvant complex [12,13].

However, measuring the protein structure and stability of antigen-protein complexes is particularly challenging due to the heterogeneous nature of the system and the low concentrations. To date, only few studies are available in the literature; in particular, circular dichroism (CD) has provided information on the changes in structure and stability of proteins bound to different types of nanoparticles [14,15].

At nanoscale, the antigens binding capacity per adjuvant mass is higher than that of traditional aluminum hydroxide-based adjuvants. This is linked to the smaller particle size, larger specific surface area, higher surface reactivity, and stronger adsorption capacity [16]. In addition, the use of nano-formulations is attracting increased interest, not only as nanomedicines [17], but also as nanovaccines [18]. For example, lipid nanoparticles carriers are used as nano-adjuvants in mRNA-based vaccines [19] against COVID-19. In fact, there is increasing evidence that adjuvants with particles in the size range of $80-150 \mathrm{~nm}$ have a more potent adjuvant activity than large microparticles [20]. In particular, nanoaluminum adjuvants, such as aluminum hydroxide nanoparticles (AH-NP), have been shown to significantly mitigate excessive inflammatory reactions (e.g., subcutaneous granuloma) at the injection site [16] and to elicit a Th1 response [21], compared to conventional aluminum hydroxide-based adjuvant. Mitigation of the inflammatory reaction could be important for clinical conditions characterized by defective immune system regulation, where particular attention on adjuvant administration should be taken [22,23].

Aiming to develop a robust characterization for nanoaluminum-subunit vaccines, we have selected nano-AH-ovalbumin as a model system. Ovalbumin (OVA) is a "classical" reference protein for immunization experiments, especially in mice [24-26]. It is a phosphorylated globular glycoprotein with 385 amino acids, molecular weight (MW) of $42.7 \mathrm{kDa}$, predicted isoelectric point (IEP) of 4.5 and a secondary structure made by $30 \% \alpha$-helix and $32 \% \beta$-sheet by $X$-ray diffraction $[27,28]$.

In this article, we propose a characterization cascade based on existing techniques for the accurate measurement of the critical physicochemical properties of nanoaluminum adjuvants and nanoadjuvant-antigen systems. This integrated experimental approach is general and is applicable to different adjuvant-antigen combinations. Its use will give a very accurate characterization of the adjuvant-antigen complex, leading to the faster development of a wide variety of much-needed vaccines. 


\section{Materials and Methods}

\subsection{Materials}

Alhydrogel ${ }^{\circledR}$ (Oxyhydroxy aluminum) was supplied by Brenntag Biosector (Essen, Germany). OVA from chicken egg and human serum albumin (HSA) were supplied by Sigma Aldrich Co., Milan, Italy.

\subsection{Preparation of Adjuvant-Antigen Complex}

Alhydrogel ${ }^{\circledR}$ was diluted from the stock to the final concentration of $1 \mathrm{mg} \mathrm{Al} / \mathrm{mL}$. An aqueous stock solution of OVA or HSA $(10 \mathrm{mg} / \mathrm{mL})$ was prepared by dissolving the protein in distilled deionized water (milli-Q $\mathrm{H}_{2} \mathrm{O}$ ), $14 \mathrm{M} \Omega$ resistance. Nanoparticles (NPs) were obtained by sonication via Vial Tweeter sonicator (UIS250v, Hielscher, Teltow, Germany) for $10 \mathrm{~min}$, intensity $0.75 \%$ and pulse every $0.5 \mathrm{~s}$. The sonicator deliver up to 5-10 watts to each sterile $1.5 \mathrm{~mL}$ vial placed in the instrument and avoids cross-contamination of samples. A series of adjuvant-antigen mass ratio was prepared (adjuvant:antigen 1:5, 1:4, $1: 3,1: 2,1: 1)$, maintaining the protein's concentration. This corresponds to $1 \mathrm{mg}$ of alum mixed with $1 \mathrm{mg}$ of the OVA protein for the 1:1 sample.

All adjuvant:antigen solutions were vigorously stirred, and complex formation was achieved in a rotating wheel for $1 \mathrm{~h}$ under a controlled temperature, mimicking the preparation of vaccine formulation for in vivo experiments.

\subsection{Particles Size, Polydispersity Index}

The average particle size and polydispersity index (PDI) were determined by dynamic light scattering (DLS), using a Zetasizer (Malvern Panalytical Ltd., Malvern, UK) at $25^{\circ} \mathrm{C}$, using a back-scattering angle of $173^{\circ}$. Measurements were performed immediately after particle dilution or preparation and each measurement was the average of 11 data sets acquired for $10 \mathrm{~s}$ each, with a $30 \mathrm{~s}$ delay between measurements. Samples were diluted 10 times to reach an attenuation factor of 7 or 8 . Measurement of particle size were performed in deionized Milli-Q water, if not differently specify.

\subsection{Surface Charge Studies: Determination of Z-Potential, Titration of Zeta-Potential against $p H$}

Z-potential values of particles were measured by electrophoretic light scattering (ELS) using a Zetasizer ZS-Nano (Malvern Instruments, Beckman Coulter, CA, USA), at $25{ }^{\circ} \mathrm{C}$. Measurements were performed immediately after particle dilution, and each measurement was the average of five data sets acquired with an automatic number of runs and 30 s delay between measurements, using the Smoluchowski fitting mode.

Titration curves (Z-potential vs. $\mathrm{pH}$ ) and isoelectric point (IEP) were measured with MPT-2 accessory of Malvern Zetasizer Nano by monitoring the change in surface charge with constant and controlled $\mathrm{pH}$ variation of the buffer. Samples were brought to acidic $\mathrm{pH}$ with $0.25 \mathrm{M} \mathrm{HCl}$ solution and then titrated with the automatic addition of $0.25 \mathrm{M} \mathrm{NaOH}$ to obtain eight experimental points in the $\mathrm{pH}$ range 3.5 to 11.4. DTS1070 or high salt cuvette were used for measures in deionized Milli-Q water and $0.9 \% \mathrm{NaCl}$, respectively.

\subsection{Particles Morphology by TEM Analysis}

Particles were characterized by transmission electron microscopy (TEM). Images were obtained using a JEOL JEM 2100 microscope (JEOL, Milan, Italy) operating at $120 \mathrm{kV}$, placing a $3 \mu \mathrm{L}$ drop of $2 \mu \mathrm{g} / \mathrm{mL}$ suspensions (AH and AH-NPs) on a Formvar Carbon coated 200 mesh copper grids (Agar Scientific, Stansted, UK), previously irradiated by Leica EM ACE200 (Leica Microsystems, Milan, Italy) for 30 s, $10 \mathrm{~mA}$ and dried in a desiccator at room temperature overnight.

\subsection{Protein Electrophoresis}

Sodium dodecyl sulphate-polyacrylamide gel electrophoresis (SDS-PAGE) was used to detect unbound protein on supernatants (SN). After $1 \mathrm{~h}$ of incubation in a rotating wheel, samples were centrifuged for $10 \mathrm{~min}$ at $10,000 \times g$ at room temperature and SN were 
collected. The same volume of SN for each sample was also loaded on Protein LabChip gel (according to manufacturer's instruction), and the presence of unbound protein was detected with Agilent Protein 80 kit with Agilent 2100 Bioanalyzer (Agilent Technologies, Santa Clara, CA, USA). The starting amount of protein, as one of the samples, was loaded as the control. The gel contains three internal controls, which allow for the quantitation of the detected protein by Agilent 2100 software (version B.02.10SI764).

\subsection{Release of Antigen from Adjuvant Particles}

Vials containing adjuvant:antigen samples at ratio $1: 2$ and $1: 1$ were incubated at $37^{\circ} \mathrm{C}$ in a thermoblock incubator, mimicking experimental in vitro conditions. A sample was withdrawn at each time point ( 24 and $48 \mathrm{~h}$ ) and centrifuged $10 \mathrm{~min}$ at $10,000 \times \mathrm{g}$ at room temperature. SN was collected and free antigen content was determined by SDS-PAGE and with the protein Bioanalyzer instrument. The percentage of antigen released was calculated considering the total protein content as $100 \%$.

\subsection{Circular Dichroism}

Vials containing the adjuvant:antigen 1:1 sample were centrifuged $10 \mathrm{~min}$, at 10,000 $\times g$ at room temperature. SN was collected and run on SDS-PAGE gel chip to confirm the absence of unbound protein in the SN. Pellets were suspended in an equal amount of Milli$\mathrm{Q} \mathrm{H}_{2} \mathrm{O}$ and diluted 25 times. $\mathrm{CD}$ spectra were acquired at $25^{\circ}$ with $\mathrm{CD}$ spectropolarimeter (JASCO Inc., Easton MD, MA, USA) equipped with a Peltier temperature-controlled cell holder. The adjuvant alone, at the same concentration as the one in the sample, was set as a baseline, while Milli- $\mathrm{Q} \mathrm{H}_{2} \mathrm{O}$ was set as the baseline for the antigen alone. Spectra were measured in quartz cuvette with $0.5 \mathrm{~cm}$ optical length. Each spectrum was obtained as an average of 4 scans, from 260 to $190 \mathrm{~nm}$ with a 1-nm bandwidth pass. Changes in protein spectra structure of the adjuvant:antigen complex, was compared to the spectra of protein alone. Data analysis was performed using the Dicroweb web-based service [29] or Bestsel software [12]. The Dichroweb spectral deconvolution was performed with the reference dataset 4 using the CDSSTR algorithm.

\section{Results and Discussion}

\subsection{Synthesis and Characterization of Nanoadjuvants}

Aluminum hydroxide particles $(\mathrm{AH})$ were obtained by diluting Alhydrogel ${ }^{\circledR}$ stock solution in Milli-Q $\mathrm{H}_{2} \mathrm{O}$ and vortexing at high speed for $1 \mathrm{~min}$. As expected, the sample obtained was composed of aggregates of $\mathrm{AH}$ fibers in the size range of 1 to $10 \mu \mathrm{m}$. Figure 1a shows a representative electron micrograph image of such aggregates, where aluminum hydroxide fibers are clearly visible. To obtain aluminum hydroxide particles in the submicron size range we considered different preparation methods normally used in the pharmaceutical sector, such as the mechanical microfluidization method and highpower sonication. In particular, we selected the vial tweeter sonicator that allows preparing samples in closed containers, thus eliminating the risk of sample contamination (or release of titanium particles) inherent with using a probe sonicator [30]. In addition, samples obtained with the vial tweeter sonicator (Figure $1 b$ ) showed a better size homogeneity (lower polydispersity index, PDI) compared to those prepared with the microfluidifier (Supplementary Materials, Table S1).

The size of AH and AH-NP were measured both by DLS and by TEM. AH-NP have a size of $180 \mathrm{~nm}$ with a PDI of 0.25 , while AH have a size of $850 \mathrm{~nm}$ and PDI of 0.19 . These values are in accordance with the electron micrographs of Figure 1 that show the presence of aggregates larger than $1 \mu \mathrm{m}$ in the case of $\mathrm{AH}$, and much smaller particles for $\mathrm{AH}-\mathrm{NP}$. 

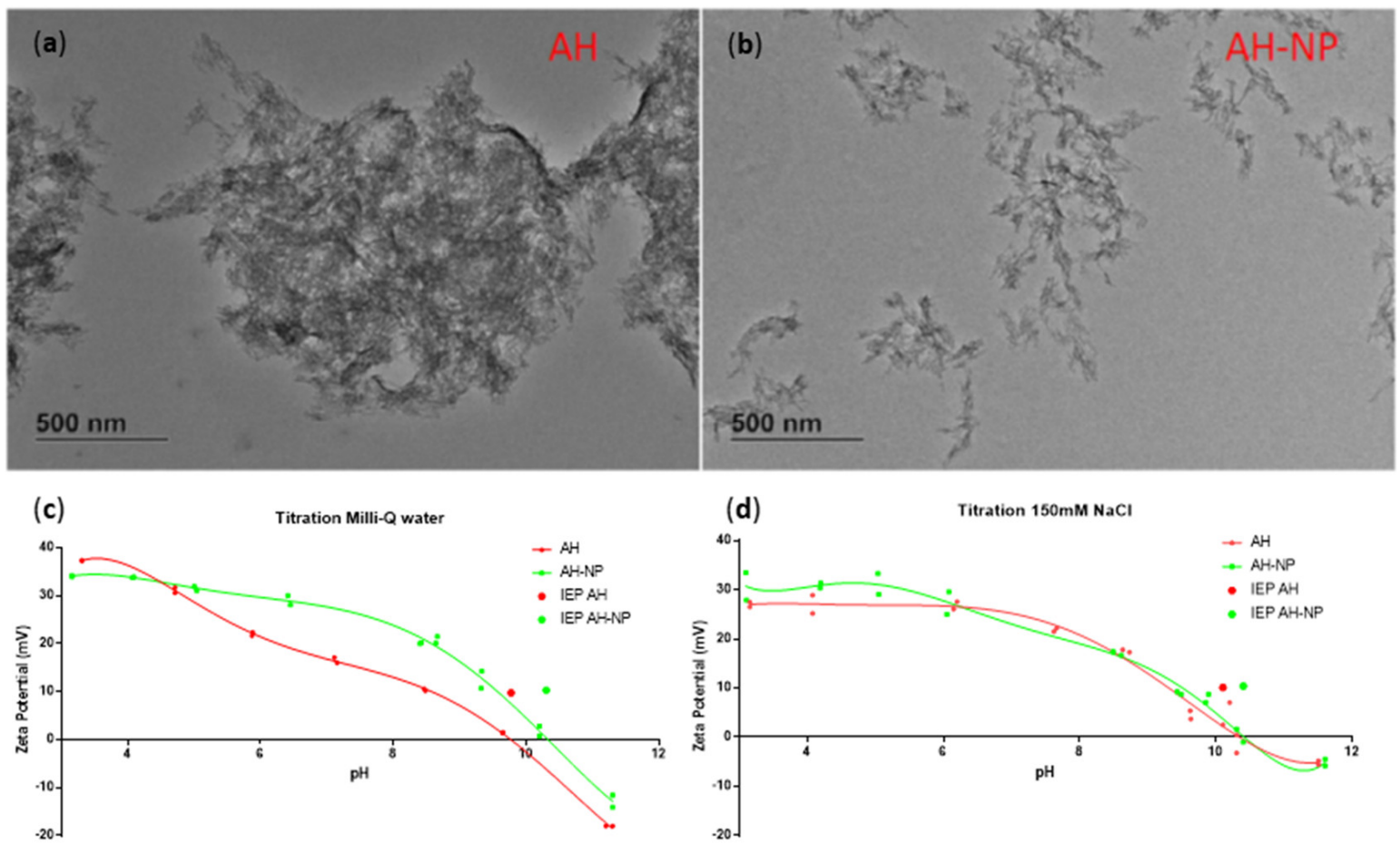

Figure 1. Characterization of AH and AH-NP. TEM analysis of (a) AH and (b) AH-NP. Z-potential vs. pH titration of AH, $\mathrm{AH}-\mathrm{NP}$ in (c) Milli-Q water and (d) isotonic $\mathrm{NaCl}(0.9 \%)$.

The surface charge of aluminum adjuvants is a key property; in fact, its value dictates the capacity of the adjuvant to bind (by electrostatic interaction) negatively charged or positively charged protein antigens. Due to their amphoteric nature, aluminum adjuvants and protein antigens, exhibit $\mathrm{pH}$-dependent charge [31]. These adjuvants can therefore change the overall surface charges based on the $\mathrm{pH}$ of the solution. Thus, the variation of the surface charge as a function of $\mathrm{pH}$ is essential to identify the best $\mathrm{pH}$ value where the adjuvant can bind a given antigen. Figure $1 c$,d shows the Z-potential of AH and AH-NP as a function of $\mathrm{pH}$. AH has an isoelectric point of around 9.8, meaning that it is positively charged (more than $+10 \mathrm{EV}$ ) at $\mathrm{pH}$ below 9.8 and negatively charged at $\mathrm{pH}$ above 10 . AH-NP has a similar isoelectric point (10.3) with a slightly different shaped Z-potential-pH curve (green curve on Figure 1c). Thus, at neutral $\mathrm{pH}$, AH-NPs are slightly more positively charged $(+25 \mathrm{mV})$ compared to $\mathrm{AH}$ adjuvant $(+15 \mathrm{mV})$.

The difference between AH and AH-NP curves, can be explained by the change of active surface (increased for AH-NP) and by the different proportion of titratable groups. In isotonic solution (Figure 1d), titration curves of both AH and AH-NP flatten, suggesting salification and fewer surface charged groups.

AH-NP tend to increase size and probably aggregate with time and temperature. Particles diluted in Roswell Park Memorial Institute (RPMI) 1640 culture media supplemented with $10 \% \mathrm{FBS}$ and kept at $37^{\circ} \mathrm{C}$ for $24 \mathrm{~h}$ showed an increase in size from $180 \mathrm{~nm}$ to $600 \mathrm{~nm}$ (Supplementary Materials, Table S2 and Figure S1). Similar increases in size were measured for AH-NP kept at $4{ }^{\circ} \mathrm{C}$ for one week. These results indicate that AH-NP has only a limited colloidal stability (as was expected from the mildly positive Z-potential) and highlight the need for using the samples immediately after preparation.

\subsection{Synthesis of Nanoadjuvant-Antigen Complexes}

We used OVA as a "classical" model antigen to develop and test the different measurements for the characterization of the adjuvant-antigen systems. The first consideration for a successful formation of aluminum adjuvant-OVA complexes is the relative charge of the two components at different $\mathrm{pH}$ values. As shown before, $\mathrm{AH}$ and $\mathrm{AH}-\mathrm{NP}$ are positively 
charged below IEP, while OVA (with predicted IEP of 5.2) is negatively charged at $\mathrm{pH}$ higher than its IEP.

The measurement of Zeta potential as a function of $\mathrm{pH}$ for OVA (Supplementary Materials, Figure S2) shows that (as expected) the protein is negatively charged at $\mathrm{pH}$ higher than 5.2. Thus, as shown in Figure 2, at neutral pH OVA is negatively charged $(-30 \mathrm{mV})$, while $\mathrm{AH}$ and AH-NP are positively charged $(+15 \mathrm{mV}$ and $+30 \mathrm{mV}$, respectively).

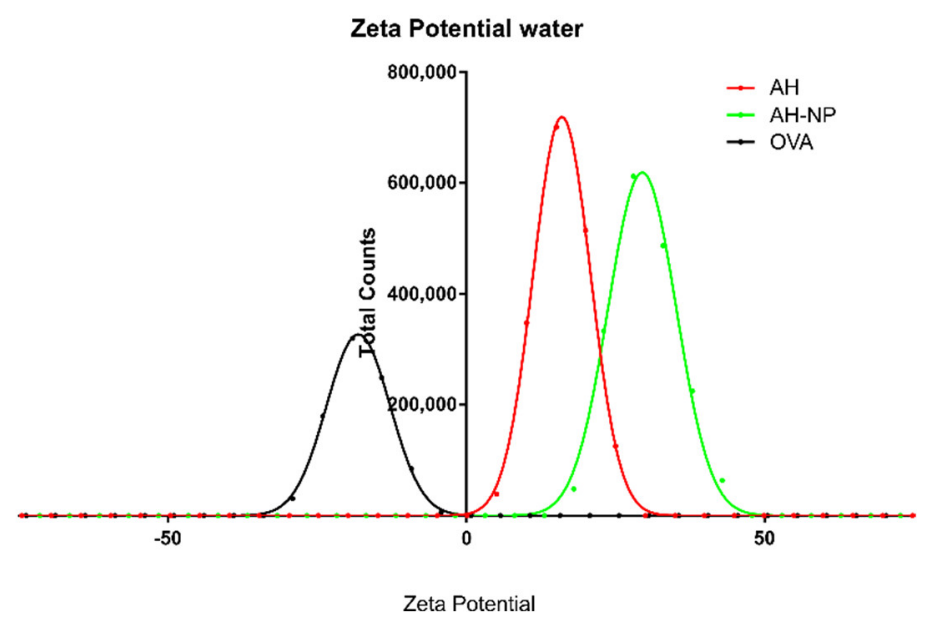

Figure 2. Zeta potential measurements of AH (red), AH-NP (green) and OVA (black) at pH 7.0.

Thus, at this $\mathrm{pH}$ there is a strong possibility that OVA binds to aluminum adjuvants via electrostatic interactions.

\subsection{Measuring Adjuvant Loading Capacity}

The relative capacity of $\mathrm{AH}$ and AH-NP to bind OVA has been tested by adding increasing amounts of the aluminum adjuvants to a constant concentration of the protein. Upon incubation and formation of the adjuvant-antigen complexes, the sample was centrifuged. The pellet (containing the complex) was removed and the amount of non-bound protein in the supernatant was measured with different techniques. In particular, the use of chip-based capillary gel electrophoresis allowed us to quantify the amounts of non-bound OVA in the supernatant with a high sensitivity and accuracy.

Figure 3 shows the amount of free OVA protein in solution as a function of the AH:OVA and AH-NP:OVA mass ratio. Data shows that, both for AH:OVA and AH-NP:OVA, all added antigen was practically bound to the adjuvant at a mass ratio of 1:2, meaning that $1 \mathrm{mg}$ of aluminum hydroxide can completely bind to $2 \mathrm{mg}$ of OVA protein. A more detailed analysis indicates that AH-NP has a slightly higher capacity to bind to the OVA antigen compared to AH. Plotting aluminum content vs. the fraction of bound OVA shows that AH-NP require around $12 \%$ less aluminum to bind the same quantity of OVA compared to $\mathrm{AH}$ (Supplementary Materials Figure S3). Making the reasonable assumption that $\mathrm{AH}$ and AH-NP have similar binding strengths, this increase in loading capacity should be due to the increased surface area available on AH-NP to bind the antigen.

The results show that in the complexes AH:OVA 1:2 and AH-NP:OVA 1:2, all the added OVA antigen became stably bound to the adjuvant, they were thus selected for the subsequent in-depth characterization. The measured surface charges of the two complexes (Z-potential) of $-6.4 \mathrm{mV}$ for AH:OVA and $-7.3 \mathrm{mV}$ for AH-NP:OVA indicated that the protein molecules likely covered the whole surface of both AH and AH-NP adjuvants (Supplementary Materials, Figure S4 showing TEM and Z-potential data). In addition, both complexes showed an increase in size compared to the adjuvants alone. AH-NP:OVA significantly increased the size from $200 \mathrm{~nm}$ to $400 \mathrm{~nm}$ and the polydispersity from 0.25 to 0.4 compared to AH-NP. AH:OVA also showed large aggregates ranging from a size of $1 \mu \mathrm{m}$ to more than $10 \mu \mathrm{m}$. Apart from the increase of size, the complexes were quite stable: 
both showed no detectable release of OVA in the supernatant after incubation for $48 \mathrm{~h}$ at $37^{\circ} \mathrm{C}$ (Supplementary Materials, Figure S5), while the size of AH-NP:OVA increased only marginally (Z average of $420 \mathrm{~nm}$ ) after $24 \mathrm{~h}$ at $37^{\circ} \mathrm{C}$.

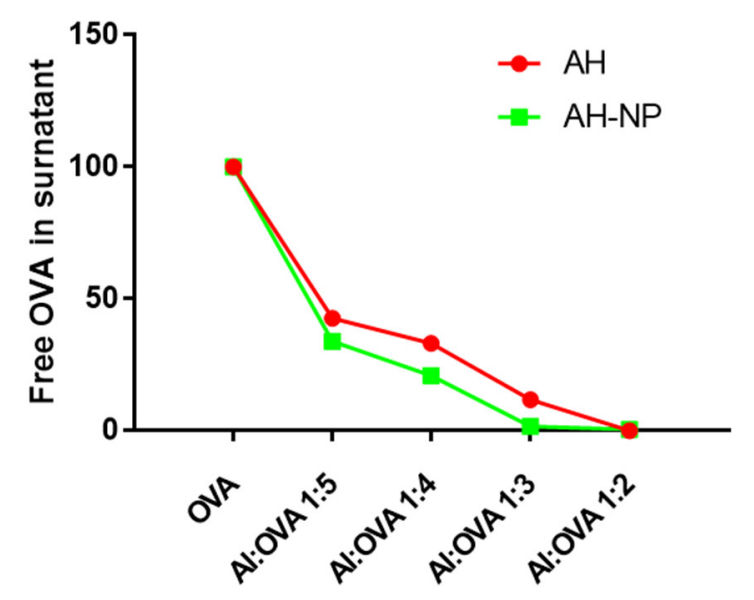

Figure 3. Chip-based capillary electrophoresis as \% of free protein in the supernatant at variable adjuvant-OVA mass ratio for AH:OVA samples (red dots) and AH-NP:OVA (green squares). Lines connecting the experimental points are for guiding the eyes only.

\subsection{Antigen Structure and Stability in Adjuvant-Antigen Complexes}

The structure of the protein antigen bound to the adjuvant is a key determinant of its immunogenicity. Protein adsorbed onto a solid surface can unfold, expose cryptic epitopes, activate the inflammatory pathways [32], and their structure can vary significantly compared to the structure in solution [9].

To date, it remains challenging to obtain structural information on proteins adsorbed on solid surfaces (such as aluminum adjuvants) [33]. Here, we used CD, a well-known biophysical technique, to measure the secondary structure changes of OVA bound to both $\mathrm{AH}$ and AH-NP adjuvants.

Figure 4 shows the CD spectra in the region 190-260 nm (sensitive to the secondary structure of proteins) of free OVA (black) and of the adjuvant-antigen samples at a mass ratio of 1:1 for both AH:OVA (red) (Figure 4a) and AH-NP:OVA(green) (Figure 4b).

The OVA CD spectra changes substantially when bound to the adjuvant surface compared to the free protein in solution. Using well-established methods for deconvoluting the CD spectra [29], it is possible to estimate the secondary structure elements of the OVA protein bound to the adjuvant and to compare to the free protein in solution. Table 1 reports the amount of each secondary structure element (helix, strand, turns, unordered) for the different samples. Upon binding to AH-NP OVA, the amount of ordered structures drastically changes, including a decrease in the $\alpha$-helix (from $74 \%$ to $16 \%$ ), and an increase in the $\beta$-sheet and turn elements (from $14 \%$ to $33 \%$ and from $8 \%$ to $24 \%$, respectively). OVA also significantly increased the amount of unordered structure (from $5 \%$ to $27 \%$ ). The changes in OVA secondary structure after binding to $\mathrm{AH}$ are smaller; the most significant is a slight decrease in $\alpha$-helix content (from $74 \%$ to $69 \%$ ) and an increase in unordered structure (from $5 \%$ to $13 \%$ ).

Table 1. Secondary structure element content of free OVA, AH-NP:OVA 1:1, AH:OVA 1:1.

\begin{tabular}{ccccc}
\hline Sample & Helix & Strand & Turns & Unordered \\
\hline Free OVA & 0.74 & 0.14 & 0.08 & 0.05 \\
AH-NP:OVA 1:1 & 0.16 & 0.33 & 0.24 & 0.27 \\
AH:OVA 1:1 & 0.69 & 0.11 & 0.05 & 0.13 \\
\hline
\end{tabular}


(a)

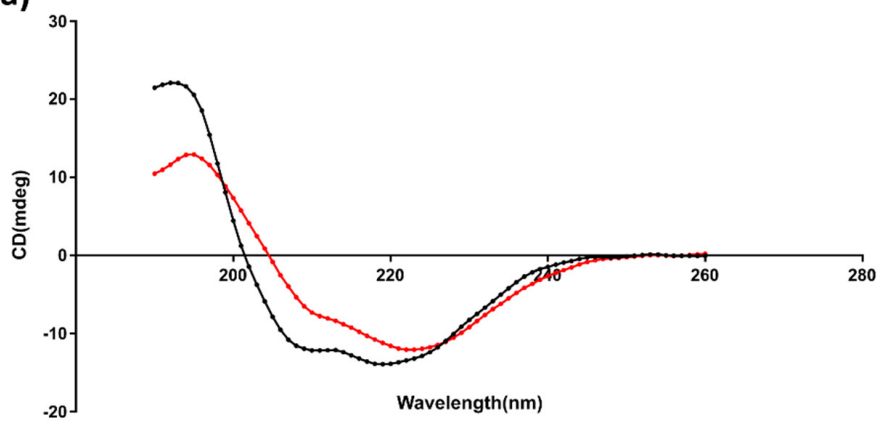

(b)

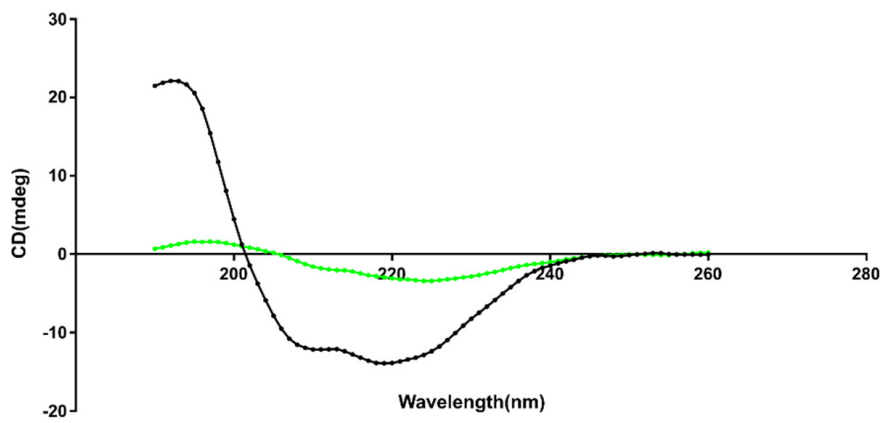

(c)

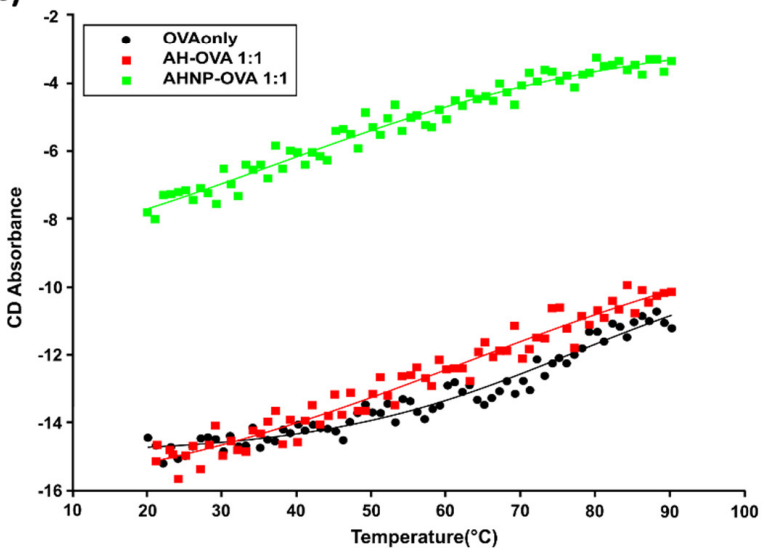

Figure 4. CD spectra of OVA bound to (a) AH or (b) AH-NP at 1:1 ratio. (c) CD thermal unfolding of free OVA, AH:OVA 1:1, AH-NP:OVA 1:1. Experimental points are shown as unconnected filled symbols (OVA: black circles; AH-OVA: red squares; AHNP-OVA: green squares). Nonlinear square fitting to Boltzman-type equation to each experimental data set as continuous lines (OVA: black; AH-OVA: red, AHNP-OVA: green).

The changes in the structure of OVA in complex with the aluminum adjuvants is a direct consequence of the protein binding to the adjuvant's surface. In fact, the experimental procedure requires the formation of the adjuvant:OVA complex, the separation of the nonadsorbed OVA by centrifugation, the control (by gel elecrophoresys) of the amount of free protein in the supernatant, and the $\mathrm{CD}$ measurement of the resuspended pellets. Our results (Supplementary Materials, Figure S5) show almost no OVA protein in the supernatant at adjuvant:antigen ratio of 1:2 and 1:1.

CD measurements of adjuvant:OVA samples allow for the determination of the changes in stability of the antigen, by measuring its thermal unfolding. Thermal unfolding was assessed by recording the intensity of the CD signal at $222 \mathrm{~nm}$ (characteristic of the $\alpha$-helical structure) as a function of the temperature (Figure $4 \mathrm{c})$. The fitting of the experimental data to a Boltzmann-type equation $\mathrm{A}+(\mathrm{B}-\mathrm{A}) /(1+\exp (\mathrm{x}-\mathrm{x} 0) / \mathrm{dx}$ (where $\mathrm{x}$ is the temperature, $\mathrm{x} 0$ the melting temperature, and $\mathrm{dx}$ the width of the thermal transi- 
tion) gave a melting temperature of $77^{\circ} \mathrm{C}$ for free OVA, $63{ }^{\circ} \mathrm{C}$ for $\mathrm{AH}: \mathrm{OVA}$ and $38^{\circ} \mathrm{C}$ for AH-NP:OVA samples.

The decrease in melting temperature for OVA adsorbed on the aluminum hydroxide adjuvants (compared to the OVA in solution) indicates a decrease in the stability of the protein bound to the adjuvant surface. These results are in good agreement with the reduction in secondary structure elements seen for OVA protein adsorbed on the adjuvant surface.

The reduction in melting temperature is much more pronounced for OVA bound to AH-NP than for OVA bound to "standard" AH.

In addition, the width of the unfolding transition $(\mathrm{dx})$ shows significant changes: it increased from $17^{\circ} \mathrm{C}$ for OVA in solution to $27^{\circ} \mathrm{C}$ and $24^{\circ} \mathrm{C}$ for AH:OVA and AH-NP:OVA, respectively. The parameter relates to the steepness of the unfolding transition and it is linked to the overall globular structure of the proteins, with the steeper unfolding being characteristic of a more globular protein folding.

The combined results of the different CD measurements (decrease in secondary structure elements, decrease in melting temperature, less steep unfolding) indicate that the OVA protein bound to the adjuvant surface has a less compact tertiary structure. This effect is much more evident for OVA bound to adjuvant nanoparticles compared to the "classical" adjuvant.

We repeated similar measurements for human serum albumin (HSA) bound to both $\mathrm{AH}$ and AH-NP adjuvants. HSA is the most abundant protein in human plasma and its availability made it suitable for studies as a model protein. It is a well-studied and characterized protein, and a carrier for endogenous and exogenous compounds delivery [34].

The analysis of CD data (Supplementary Materials, Figure S6, and Table S3) shows a decrease in $\alpha$-helical content (from $80 \%$ to $60 \%$ and $55 \%$ for HSA bound to AH and AH-NP, respectively). The CD-detected thermal unfolding (Supplementary Materials, Figure S7) shows a decrease in melting temperature (from $76{ }^{\circ} \mathrm{C}$ for HSA in solution to $61{ }^{\circ} \mathrm{C}$ and $45^{\circ} \mathrm{C}$ for AH-HSA 1:1 and AHNP-HSA 1:1, respectively) for HSA adsorbed on the adjuvant surface. In addition, there is a significantly less steep unfolding ( $\mathrm{dx}$ from $10{ }^{\circ} \mathrm{C}$ for $\mathrm{HSA}$ in solution to $19^{\circ} \mathrm{C}$ and $18{ }^{\circ} \mathrm{C}$ for AH-HSA 1:1 and AHNP-HSA 1:1, respectively).

These results are similar to those obtained for OVA: the HSA bound to the adjuvants has a lower secondary structure content, lower thermal unfolding, and less steep unfolding compared to HSA in solution. This indicates that also HSA bound to the adjuvants has a less compact tertiary structure and reduced stability. In addition, these effects are more pronunced in the case of AH-NP compared to the classical AH adjuvant, in a similar fashion as seen for OVA bound to AH-NP and classic AH. Previous work using scanning calorimetry measurements has shown a reduced thermal stability of the protein antigen when adsorbed onto aluminum salt [13]. This phenomenon could facilitate the presentation of the antigen, and is thus considered one of the mechanisms of adjuvanticity of aluminum adjuvants [13].

According to a recent model for the influence of protein fold stability on the availability of T- and B-cell epitopes, each protein has an optimal conformational stability for immunogenicity [9]. Protein antigens at the two extremes of fold stability would result in reduced immunogenicity: highly destabilized proteins are degraded too early, while hyperstabilized proteins undergo a too-slow proteolytic degradation, resulting in reduced antibody responses [9].

Our results show that adjuvants slightly reduce the stability for both OVA and HSA and this effect is more pronounced with AH-NP compared to AH. Overall, this indicates that the binding of proteins to aluminum adjuvants modulates the fold stability of the antigens. It is a reasonable working hypothesis that the absorption of protein antigens on aluminum adjuvant slightly reduces the conformational stability of the protein, leading to an increase in the exposure of T- and B-cell epitopes, which could contribute to the higher immunogenicity of protein-antigens systems compared to protein alone.

In addition, the method developed here can be used with any protein-bound to aluminum adjuvants, thus allowing to directly measure the conformational stability of any 
protein adsorbed on the adjuvant surface. This will provide an experimental technique to now assess the modulation of antigen fold stability in different vaccine formulations. For example, it will be possible to correlate the aging of some aluminum-based subunit vaccines with enhanced immune response, as in the case of the Diptheria toxoid vaccine [35].

\subsection{Physicochemical Characterization Cascade for Nanoadjuvant-Antigen Systems}

Accurate characterization of physicochemical properties of adjuvants is essential for developing more effective vaccines; for example, as shown here, to identify the $\mathrm{pH}$ values where antigen and adjuvant maximize their interaction. Here we have shown that using a systematic approach, making use of the different existing techniques developed and applied in various scientific fields (such as nanotechnology, biotechnology, and biochemistry) it is possible to synthesize nanoadjuvant-antigen complexes and to accurately measure their key properties. Figure 5 summarized the process we developed and employed in this work. It can be used as a general, stepwise characterization cascade for an accurate physicochemical characterization of nanoadjuvant-antigen systems.

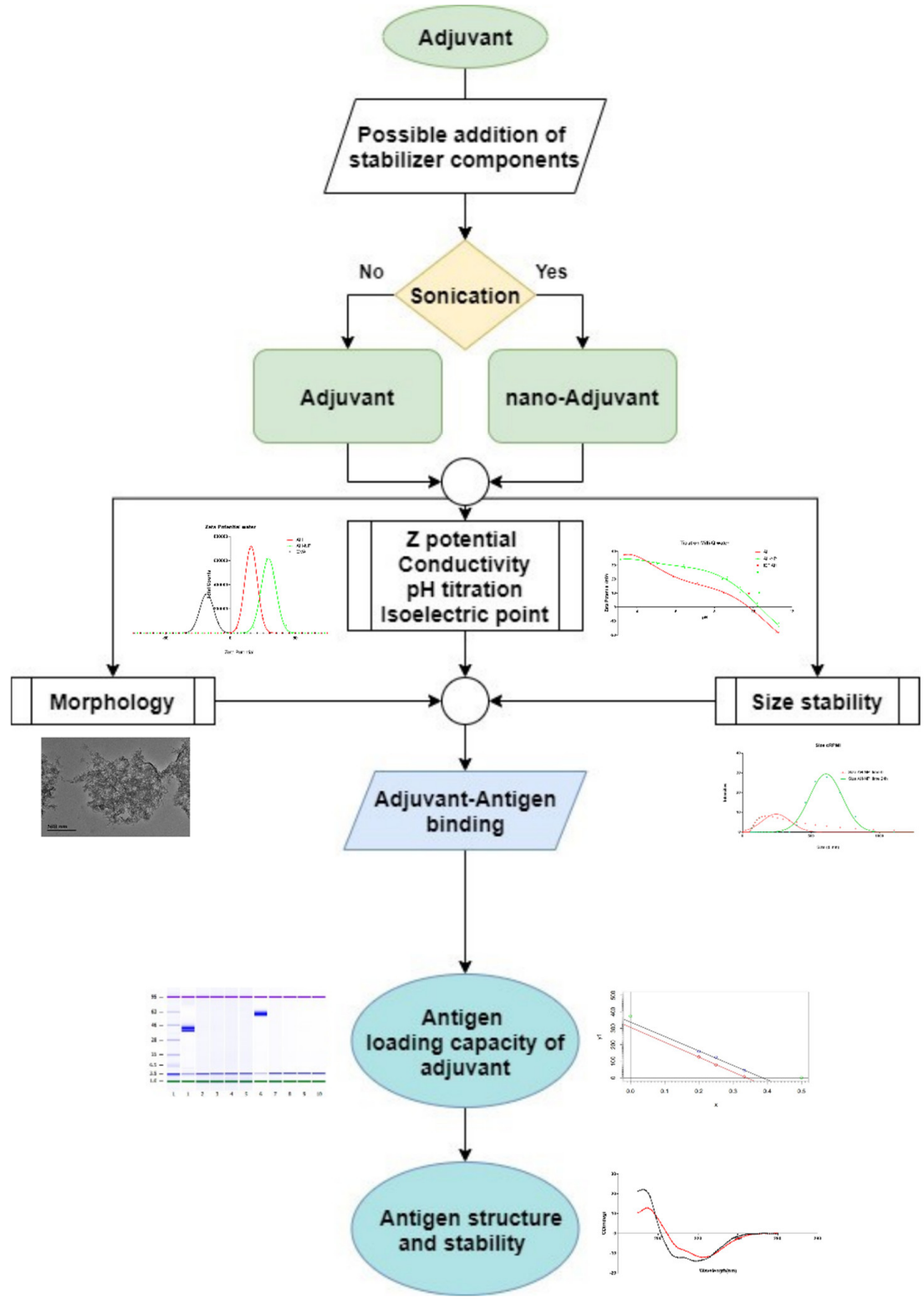

Figure 5. Schematic overview of the physicochemical characterization of nanoadjuvant-protein antigen systems used in this study. 
Following the identification of the most appropriate adjuvant (and eventually stabilizers), the first step consists of the preparation of the nanoadjuvant, its basic properties, average size, and electrostatic properties, which are determined. Measuring the Z-potential at different $\mathrm{pH}$ values allows determining its isoelectric point and estimate its colloidal stability. More in-depth analysis is needed to measure the stability in relevant physiological buffers (aggregation propensity with DLS), while the morphology of the nanoadjuvant is determined by electron microscopy.

The obtained information allows determining the best $\mathrm{pH}$ for the formation of the antigen-adjuvant complex. Another fundamental property is the antigen-loading capacity of the adjuvant; this can be measured with high sensitivity and accuracy by first separating the complex by centrifugation and then measuring the amount of adjuvant-bound protein by digital electrophoresis.

Finally, the structure and stability of the antigen in the antigen-adjuvant complex is analysed by CD. These measurements allow to directly quantify the changes in fold stability of the antigen adsorbed on the adjuvant surface and eventually to modulate it (by altering the formulation) to improve the immune response.

This characterization cascade has been applied to the nanoaluminum-OVA system (and confirmed using nanoaluminum-HSA), it has the advantage that can be applied to almost any combination of nanoadjuvant-antigen system.

\section{Conclusions}

The accurate measurements of the physicochemical properties of adjuvants and adjuvant-antigen systems is of critical importance for the rational design of vaccine formulation. The characterization cascade presented here follows a stepwise approach of increased complexity, starting from the nanoadjuvant alone, up to the very challenging measurement of the structure of the antigens adsorbed on the adjuvant. In particular, the use of the $\mathrm{CD}$ technique allows for direct measurement of the structural stability of the antigen adsorbed on the aluminum adjuvant surface. This allows for a direct and simple experimental correlation between the immunogenicity and fold stability of the adsorbed antigens in different vaccine formulations.

This characterization cascade has been developed and tested for nanoaluminum-OVA (and HSA) protein antigens but generally, it can be easily applied to any adjuvant-antigen combination. It will contribute to the development of better antigen-adjuvant complexes and immunological outcomes, ultimately leading to improved vaccines.

Supplementary Materials: The following are available online at https:/ / www.mdpi.com/article/ 10.3390/vaccines9060544/s1, Figure S1: Change in the size of AH-NP incubated in RPMI culture media, Figure S2: Measurement of Zeta potential as a function of $\mathrm{pH}$ for ovalbumin, Figure S3: Plot of Aluminum mass vs amount of free antigen, Figure S4: TEM micrograph of AH:OVA 1:2 complex, Figure S5: Chip-based capillary electrophoresis, Figure S6: Secondary structure of HSA bound to AH or AH-NP, Figure S7: CD thermal unfolding of free HSA, AH:HSA 1:1, AH-NP:HSA 1:1, Table S1: Comparison with different methods of aluminum nano-particles preparation, Table S2: DLS of AH-NP at time 0 and after $24 \mathrm{~h}$ at $37^{\circ} \mathrm{C}$, Table S3: Secondary structure elements content of free HSA, AH:HSA 1:2, AH-NP:HSA 1:2.

Author Contributions: Conceptualization, G.G., A.V., A.H., D.M. (Donata Medaglini) and L.C.; Investigation, G.G., A.V., S.G., J.P., D.M. (Davide Magrì), D.M. (Donata Medaglini) and L.C.; Writingoriginal draft, G.G., A.V., D.M. (Donata Medaglini) and L.C.; Writing-review \& editing, S.G., J.P., D.M. (Davide Magrì) and A.H. All authors have read and agreed to the published version of the manuscript.

Funding: This research was partly funded by the EC Joint Research Centre Exploratory Research program under the project "NanoMicrobials" and by the EC Seventh Framework Programme under the project ADITEC (grant number HEALTH-2011-280873) and Horizon 2020 Framework Programme under the project TRANSVAC (grant number 730694).

Conflicts of Interest: The authors declare no conflict of interest. 


\section{References}

1. HogenEsch, H.; O'Hagan, D.T.; Fox, C.B. Optimizing the Utilization of Aluminum Adjuvants in Vaccines: You Might Just Get What You Want. npj Vaccines 2018, 3, 51. [CrossRef]

2. Adjuvants and Vaccines I Vaccine Safety ICDC. Available online: https://www.cdc.gov/vaccinesafety/concerns/adjuvants.html (accessed on 14 January 2021).

3. HogenEsch, H. Mechanism of Immunopotentiation and Safety of Aluminum Adjuvants. Front. Immunol. 2013, 3, 51. [CrossRef]

4. Draft Landscape of COVID-19 Candidate Vaccines. Available online: https://www.who.int/publications/m/item/draftlandscape-of-covid-19-candidate-vaccines (accessed on 20 April 2021).

5. O'Hagan, D.T.; Lodaya, R.N.; Lofano, G. The Continued Advance of Vaccine Adjuvants—'We Can Work It Out'. Semin. Immunol. 2020, 50, 101426. [CrossRef] [PubMed]

6. Sokolovska, A.; Hem, S.L.; HogenEsch, H. Activation of Dendritic Cells and Induction of CD4+ T Cell Differentiation by Aluminum-Containing Adjuvants. Vaccine 2007, 25, 4575-4585. [CrossRef]

7. Marrack, P.; McKee, A.S.; Munks, M.W. Towards an Understanding of the Adjuvant Action of Aluminium. Nat. Rev. Immunol. 2009, 9, 287-293. [CrossRef]

8. Clapp, T.; Siebert, P.; Chen, D.; Jones Braun, L. Vaccines with Aluminum-Containing Adjuvants: Optimizing Vaccine Efficacy and Thermal Stability. J. Pharm. Sci. 2011, 100, 388-401. [CrossRef] [PubMed]

9. Scheiblhofer, S.; Laimer, J.; Machado, Y.; Weiss, R.; Thalhamer, J. Influence of Protein Fold Stability on Immunogenicity and Its Implications for Vaccine Design. Expert Rev. Vaccines 2017, 16, 479-489. [CrossRef] [PubMed]

10. Iyer, V.; Hu, L.; Liyanage, M.R.; Esfandiary, R.; Reinisch, C.; Meinke, A.; Maisonneuve, J.; Volkin, D.B.; Joshi, S.B.; Middaugh, C.R. Preformulation Characterization of an Aluminum Salt-Adjuvanted Trivalent Recombinant Protein-Based Vaccine Candidate Against Streptococcus Pneumoniae. J. Pharm. Sci. 2012, 101, 3078-3090. [CrossRef] [PubMed]

11. Régnier, M.; Metz, B.; Tilstra, W.; Hendriksen, C.; Jiskoot, W.; Norde, W.; Kersten, G. Structural Perturbation of Diphtheria Toxoid upon Adsorption to Aluminium Hydroxide Adjuvant. Vaccine 2012, 30, 6783-6788. [CrossRef]

12. Micsonai, A.; Wien, F.; Bulyáki, É.; Kun, J.; Moussong, É.; Lee, Y.-H.; Goto, Y.; Réfrégiers, M.; Kardos, J. BeStSel: A Web Server for Accurate Protein Secondary Structure Prediction and Fold Recognition from the Circular Dichroism Spectra. Nucleic Acids Res. 2018, 46, W315-W322. [CrossRef]

13. Jones, L.S.; Peek, L.J.; Power, J.; Markham, A.; Yazzie, B.; Middaugh, C.R. Effects of Adsorption to Aluminum Salt Adjuvants on the Structure and Stability of Model Protein Antigens. J. Biol. Chem. 2005, 280, 13406-13414. [CrossRef]

14. Capomaccio, R.; Jimenez, I.O.; Colpo, P.; Gilliland, D.; Ceccone, G.; Rossi, F.; Calzolai, L. Determination of the Structure and Morphology of Gold Nanoparticle-HSA Protein Complexes. Nanoscale 2015, 7, 17653-17657. [CrossRef] [PubMed]

15. Laera, S.; Ceccone, G.; Rossi, F.; Gilliland, D.; Hussain, R.; Siligardi, G.; Calzolai, L. Measuring Protein Structure and Stability of Protein-Nanoparticle Systems with Synchrotron Radiation Circular Dichroism. Nano Lett. 2011, 11, 4480-4484. [CrossRef]

16. He, P.; Zou, Y.; Hu, Z. Advances in Aluminum Hydroxide-Based Adjuvant Research and Its Mechanism. Hum. Vaccin Immunother. 2015, 11, 477-488. [CrossRef]

17. Farjadian, F.; Ghasemi, A.; Gohari, O.; Roointan, A.; Karimi, M.; Hamblin, M.R. Nanopharmaceuticals and Nanomedicines Currently on the Market: Challenges and Opportunities. Nanomedicine 2019, 14, 93-126. [CrossRef]

18. Singh, A. Eliciting B Cell Immunity against Infectious Diseases Using Nanovaccines. Nat. Nanotechnol. 2021, 16, 16-24. [CrossRef] [PubMed]

19. Kowalski, P.S.; Rudra, A.; Miao, L.; Anderson, D.G. Delivering the Messenger: Advances in Technologies for Therapeutic MRNA Delivery. Mol. Ther. 2019, 27, 710-728. [CrossRef] [PubMed]

20. Li, X.; Aldayel, A.M.; Cui, Z. Aluminum Hydroxide Nanoparticles Show a Stronger Vaccine Adjuvant Activity than Traditional Aluminum Hydroxide Microparticles. J. Control Release 2014, 173, 148-157. [CrossRef]

21. Orr, M.T.; Khandhar, A.P.; Seydoux, E.; Liang, H.; Gage, E.; Mikasa, T.; Beebe, E.L.; Rintala, N.D.; Persson, K.H.; Ahniyaz, A.; et al. Reprogramming the Adjuvant Properties of Aluminum Oxyhydroxide with Nanoparticle Technology. npj Vaccines 2019, 4, 1-10. [CrossRef]

22. Murdaca, G.; Orsi, A.; Spanò, F.; Puppo, F.; Durando, P.; Icardi, G.; Ansaldi, F. Influenza and Pneumococcal Vaccinations of Patients with Systemic Lupus Erythematosus: Current Views upon Safety and Immunogenicity. Autoimmun. Rev. 2014, 13, 75-84. [CrossRef] [PubMed]

23. Watad, A.; Quaresma, M.; Bragazzi, N.L.; Cervera, R.; Tervaert, J.W.C.; Amital, H.; Shoenfeld, Y. The Autoimmune/Inflammatory Syndrome Induced by Adjuvants (ASIA)/Shoenfeld's Syndrome: Descriptive Analysis of 300 Patients from the International ASIA Syndrome Registry. Clin. Rheumatol. 2018, 37, 483-493. [CrossRef] [PubMed]

24. Mahony, D.; Cavallaro, A.S.; Stahr, F.; Mahony, T.J.; Qiao, S.Z.; Mitter, N. Mesoporous Silica Nanoparticles Act as a Self-Adjuvant for Ovalbumin Model Antigen in Mice. Small 2013, 9, 3138-3146. [CrossRef]

25. Kim, J.H.; Ohsawa, M. Oral Tolerance to Ovalbumin in Mice as a Model for Detecting Modulators of the Immunologic Tolerance to a Specific Antigen. Biol. Pharm. Bull. 1995, 18, 854-858. [CrossRef]

26. Courtenay, A.J.; Rodgers, A.M.; McCrudden, M.T.C.; McCarthy, H.O.; Donnelly, R.F. Novel Hydrogel-Forming Microneedle Array for Intradermal Vaccination in Mice Using Ovalbumin as a Model Protein Antigen. Mol. Pharm. 2019, 16, 118-127. [CrossRef] [PubMed] 
27. Chay Pak Ting, B.P.; Pouliot, Y.; Gauthier, S.F.; Mine, Y. 19-Fractionation of egg proteins and peptides for nutraceutical applications. In Separation, Extraction and Concentration Processes in the Food, Beverage and Nutraceutical Industries; Rizvi, S.S.H., Ed.; Woodhead Publishing Series in Food Science, Technology and Nutrition; Woodhead Publishing: Sawston, UK, 2013; pp. 595-618, ISBN 9781845696450.

28. Huntington, J.A.; Stein, P.E. Structure and Properties of Ovalbumin. J. Chromatogr. B Biomed. Sci. Appl. 2001, 756, 189-198. [CrossRef]

29. Whitmore, L.; Wallace, B.A. DICHROWEB, an Online Server for Protein Secondary Structure Analyses from Circular Dichroism Spectroscopic Data. Nucleic Acids Res. 2004, 32, W668-W673. [CrossRef]

30. Betts, J.N.; Johnson, M.G.; Rygiewicz, P.T.; King, G.A.; Andersen, C.P. Potential for Metal Contamination by Direct Sonication of Nanoparticle Suspensions. Environ. Toxicol. Chem. 2013, 32, 889-893. [CrossRef] [PubMed]

31. Al-Shakhshir, R.; Regnier, F.; White, J.L.; Hem, S.L. Effect of Protein Adsorption on the Surface Charge Characteristics of Aluminium-Containing Adjuvants. Vaccine 1994, 12, 472-474. [CrossRef]

32. Deng, Z.J.; Liang, M.; Monteiro, M.; Toth, I.; Minchin, R.F. Nanoparticle-Induced Unfolding of Fibrinogen Promotes Mac-1 Receptor Activation and Inflammation. Nat. Nanotechnol. 2011, 6, 39-44. [CrossRef]

33. Calzolai, L.; Laera, S.; Ceccone, G.; Rossi, F. Structure and Stability of Proteins Interacting with Nanoparticles. In Proteins at Interfaces III State of the Art; ACS Symposium Series; American Chemical Society: Washington, DC, USA, 2012; Volume 1120, pp. 839-855, ISBN 9780841227965.

34. Wang, Y.; Wang, S.; Huang, M. Structure and Enzymatic Activities of Human Serum Albumin. Curr. Pharm. Des. 2015, 21, 1831-1836. [CrossRef]

35. Gupta, R.K. Aluminum Compounds as Vaccine Adjuvants. Adv. Drug Deliv. Rev. 1998, 32, 155-172. [CrossRef] 